\title{
Prevalence of Dissociative Experiences in Those Referred to Emergency Psychiatric Centers After Attempting Suicide
}

\author{
Ali Firoozabadi', Leila Razeghian Jahromi', Shokoofeh Yaghmaie ${ }^{1 *}$ \\ ${ }^{1}$ School of Medicine, Shiraz University of Medical Sciences, Shiraz, Iran \\ *Corresponding Author: Shokoofeh Yaghmaie, M.D., School of Medicine, Shiraz University of Medical Sciences, \\ Shiraz, Iran. Tel: +98-9171099401, Email: shokooofeye_shokooofan@yahoo.com
}

Received October 5, 2017; Accepted December 10, 2017; Online Published February 10, 2018

\begin{abstract}
Background: Dissociation is a symptom that can be related to traumatic childhood events. Dissociation in some cases is categorized in a distinct subgroup from other psychiatric disorders.

Objective: The purpose of this study was to investigate the prevalence of dissociative experiences in patients who have attempted suicide and who have referred to an emergency psychiatric center.

Methods: This was a cross-sectional epidemiological study in which dissociative experiences were evaluated in 98 patients who referred to Ibn Sina and Hafez hospitals after attempting suicide. In addition to determining the prevalence of these experiences, the relation between the symptoms and variables such as sex, age, marital status, education and suicide risk was determined.

Results: There was a significant difference in the level of disappointment between married and single patients $(P=0.047)$. The mean disappointment score for the overall population was 11.92, which is in the normal range. There was no significant relationship between the dissociation score and level of disappointment $(P=0.933)$. The prevalence of dissociative experiences was found to decrease as the age of the patients increased $(P=0.006)$. There was no significant difference between the rate of suicide as reflected in the measurement of disappointment and dissociative symptoms. There was no significant relationship between DES score and other variables.

Conclusion: One cause of psychological pressure in deciding to attempt suicide is family conflict. Many individuals who attempt suicide did not have a thought-out desire to take their lives, but attempted it impulsively in response to a periodic stressor.

Keywords: Dissociation Disorders, Suicide, Disappointment, Traumatic Experience
\end{abstract}

\section{Introduction}

Dissociative disorders involve a disruption or breakdown originating in the surrounding environment that can divide the emotions and body of an individual. These may occur suddenly or gradually and be transient or chronic. ${ }^{1}$ Unlike psychosis, the most important characteristic of dissociative disorders is a rupture from reality. ${ }^{2-5} \mathrm{~A}$ dissociative experience is a defense mechanism against crisis or exposure to stress. ${ }^{6-8}$ The DSM-IV-TR identifies the types of dissociative disorders as dissociative identity, personality deprivation, dissociative forgetfulness and unspecified dissociative disorders. ${ }^{9}$ One consequence of suicide stemming from mental, physical and sexual abuse is a dissociative disorder. ${ }^{10-12}$ Dissociative disorders have high specificity and low sensitivity to a traumatic experience. There is a history of trauma in a large number of patients with dissociative disorders; however, many with a traumatic history do not have symptoms of this disorder. ${ }^{13}$ In those affected, the signs of a dissociative disorder may manifest as a depersonalization disorder, forgetfulness and psychological anesthesia. In severe trauma, these shortterm symptoms can be effective defense mechanisms, but in the long run, they decrease the ability of the individual to function. ${ }^{14}$ Attempting suicide at a younger age has been associated with a high level of dissociative disorder. ${ }^{15}$

Dissociation was initially considered to be an adaptive response to the pain and fear of mental harm. If it continues over time, it can retard personality development. It is the continuous process of integrating memories and understanding one's self and the perceptions and emotions of other people. ${ }^{2}$

Stronger dissociation in memory and identity

Copyright (C 2018 The Author(s). This is an open-access article distributed under the terms of the Creative Commons Attribution License (http:// creativecommons.org/licenses/by/4.0), which permits unrestricted use, distribution, and reproduction in any medium, provided the original work is properly cited. 
(forgetfulness, escape) can be demonstrated by dissociative defense. Ultimately, this mechanism can lead to the formation of independent characteristics that appear in the form of multiple-personality disorder. Organization of memories depends on the establishment of continuity between the encryption, storage and retrieval sectors. For successful remembrance, episodic memories should be encrypted in a timely manner and stored in such a way that they can be remembered with specific configurations. Chronic forgetfulness and dissociation occur in response to severe mental harm, such as war, rape and torture. However, in some cases, the social environment itself is not harmful, but is highly conflicted. The patient experiences unbearable feelings such as embarrassment, guilt, disappointment, anger and frustration. In response to conflicts, when a response to war or escape is not possible, memory loss can be a substitute for suicide. ${ }^{2}$

Most victims face a communication upheaval. Because the abusive agent is often the same person who is responsible for the individual's care, in order to maintain affection, the reality of mistreatment must be denied, forgotten, suppressed and marginalized. This is a dilemma and the associated defense mechanisms that do more than harm provide the scene for the emergence and development of dissociative modes. Memory is an active process coupled with rebuilding and is affected by incitement and information from a variety of sources. Mental damage affects tacit and explicit memory differently. The non-verbal aspects of the experience, such as a fear response, can be implicitly encoded, although the verbal aspects may not be encrypted. This is related to the hippocampus during the period of mental injury. The physiological, emotional, perceptual and motor aspects of mental harm, in particular anxiety and fear responses, are encoded in other areas, particularly the amygdala (which, unlike the hippocampus, is not affected by stress hormones). ${ }^{1-5}$ Therefore, the traumatic experience cannot be reminiscent of fictional and verbal forms, while physical memory associated with it continues to be in the form of fears and psychological problems.

Traumatic experience can never enter into autobiographical memory. The history of physical, verbal and sexual mistreatment has been observed in disorders and in all psychiatric disorders. The response to childhood psychological injury can be primary or secondary. Initial responses are the direct result of dealing with injury and secondary responses are problems that a person encounters during attempts to cope with these initial effects. Studies have shown that at least three psychiatric disorders are the direct consequence of conflict with severe childhood injury: post-traumatic stress disorder, dissociative disorders (including alteration disorder) and borderline personality disorder. The second category includes disorders such as drug addiction, eating disorders, physical impairment, hypochondria and obsessive disorder. ${ }^{1}$

Several studies have suggested a relationship between suicidal thoughts and self-harm tendencies on the one hand and dissociative experiences on the other. Self-harm is a way to cope with unbearable emotions that result from severe childhood injury. Screening studies show that $10 \%$ of dissociative experiences in the United States occur in psychiatric patients, addicts and prostitutes. ${ }^{3}$

The term "dissociation" refers to a break in the natural and coherent experiences of the individual. The American Psychiatric Association DSM-IV-TR defines the role played by dissociation disorders as "a breakdown in the normally consistent functions of consciousness, memory, identity and perception". ${ }^{3}$ In the fifth edition (DSM-V), motion control, behavior and physical manifestations have been added to the list of functions, which approaches the International Classification of Diseases definition of dissociation phenomenon. ${ }^{4}$

A break in coherent functions has adaptive and maladaptive consequences. On the one hand, it gives the person a chance to pull himself out of the undercurrent of psychological stress and, on the other hand, if it is prolonged, it will lead to disruption of normal functioning. Dissociation is the division of an experience into parts which are not integrated into a unified and continuous totality, but are stored separately. Dissociation is a form of organization to experience.

\section{Objective}

The aim of this study was to evaluate the prevalence of dissociative experiences in patients referring to the emergency psychiatric center of Ibn Sina and Ali Asghar hospitals in Shiraz after a suicide attempt. The goal is to help researchers and therapists to better understand those who attempt suicide and the underlying issues that have caused the crisis in their lives.

\section{Methods}

A total of 100 patients referring to the psychiatric emergency centers of the hospitals were selected through easy sampling (periodical). The patients should not have been exposed to narcotic drugs at the time of the study or have had active psychosomatic symptoms and must have no history of drug addiction. Two such patients were thus excluded from the statistical population.

The sampling method was easy sampling of available cases. A prevalent assumption about dissociative experiences is that they actually form a spectrum from mildly experiential and natural aspects of daily life to a marked psychiatric disorder such as identity dissociative disorder. ${ }^{16}$ The questionnaire developed by Bernstein and Putnam for analyzing patient experience was used in the current study. ${ }^{17}$ It consists of 28 questions and has a global and independent culture that has been used by various communities to evaluate dissociative experiences with high validity. Some researchers have argued that natural and illness dissociation show qualitative differences somewhere in this range and they speak of a group of people who have inherent and intrinsic vulnerability to dissociation.

The questions ask individuals to assess their degree 
of experience from zero to 100 . The total score of an individual is the sum of these scores divided by 28 . It has been observed that a score higher than 30 is greatly predictive of severe psychiatric disorders, especially multiple-personality disorder. This questionnaire has been used more than any other questionnaire in studies related to dissociation. It is a screening questionnaire and is not used to diagnose the disorder. The average score in the general population of the United States is 10.8 , a score lower than 15 is considered as normal, and of more than 15 is assessed as:

20-15: slightly above normal

30-20: high with a probability of PTSD or DDNOS

40-30: extremely high probability of DID

40 or higher: increased likelihood of DID

A high score indicates a higher probability of a dissociative disorder. This questionnaire is widely used for assessment of dissociative symptoms globally and shows a high convergence with other questionnaires used to assess dissociative disturbances. It also has a high predictive value for identity dissociative disorder, traumatic experience and mistreatment history. The reliability of this questionnaire was determined after its translation into Farsi and subsequent retranslation into English. Its validity was investigated using 30 medical students over a 2-week period and showed a Cronbach a of 0.78 .

The level of individual disappointment, or the rate of suicidal inclination, was measured using Beck's hopelessness scale. The questionnaire contains 20 questions with true and false options. Questions 1, 6, 13, 15 and 19 measure the individual's perception of the future. Questions 2, 3, 9, $11,12,16,17$ and 20 assess lack of stimulation. Questions 4, 7, 8, 14 and 18 measure hope and expectation about the future. Questions 2, 4, 7, 9, 11, 12, 14, 16, 17, 18, and 20 will earn one point for the true option and questions $1,3,5,6$, $8,10,13,15$ and 19 will do so for the false option.

The total score of each person will fall between 0 and 20. The interpretation is as follows: a score of less than 3 is low hopelessness; 4 to 8 is somewhat disappointed; 9 to 14 is moderately and significantly disappointed and greater than or equal to 15 is severe disappointment. SPSS software and analysis of variance (ANOVA) were used to analyze the results. The DES scores were classified as: $15=$ normal, 16 to $20=$ mild; 21 to $30=$ medium; over $30=$ severe .

\section{Results}

Of the 98 subjects, 40 (40.8\%) were male and 58 (59.2\%) were female. A total of $26(28.5 \%)$ were single and 72 (71.5\%) were married. For educational level, 28 (28.6\%) had intermediate school education and 40 (40.8\%) had a high school diploma; 30 (30.6\%) had an undergraduate degree or were pursuing a graduate degree. Of the total, 11 (11.2\%) were unemployed; 17 (17.3\%) were employed; 24 (24.5\%) were self-employed; 5 (5.1\%) were retired; 7 (7\%) were students and $34(34.7 \%)$ were housewives.

The number of patients expressing a level of disappointment was rated on the basis of Beck's hopelessness scale: slightly disappointed: (none); somewhat disappointed: 13 (13.03); moderately disappointed: 67 (68.4); highly disappointed:18 (18.4). Only $18.4 \%$ of patients showed high disappointment (18 persons: 10 male and 8 female; 5 housewives, 2 employed, 2 students, 8 self-employed, 1 retired); educational level completed: 4 intermediate school, 7 high school diploma, 5 undergraduate degrees, 2 master's degrees).

The prevalence of dissociative experiences in the patients was as follows: 44 people $(44.9 \%)$ scored 15 or less; $15(15.3 \%)$ scored $15+$ to 20,17 (17.3\%) scored $20+$ to 30 and $22(22.4 \%)$ scored over 30 . The mean DES score was 21.7 , which did not differ significantly between males and females. The DES scores were divided into married and single groups. There was no significant difference between groups for dissociation score $(P=0.139)$. The independent $\mathrm{t}$-test results were presented to compare the degree of disappointment between the married and single groups. There was a significant difference between the average disappointment of the two groups $(P=0.047)$. Independent $t$ test results were presented to compare the mean DES score of males and females and no significant difference was determined between these groups $(P=$ $0.064)$.

One-way ANOVA showed a significant relationship between the mean DES score and individual occupation $(P$ $=0.027)$. There was a significant relationship with employed $(P=0.017)$ and self-employed $(P=0.013)$, but there was no significant relationship with housewife, retired or students. Being employed had significant relationship with being a housewife $(P=0.03)$. Being self-employed had a significant relationship with being a housewife $(P=0.018)$. Retirement and student status showed no significant relationships.

No significant correlation was found between disappointment and dissociative experiences, which means that neither affected the other $(P=0.933)$. The Pearson correlation test indicated a significant correlation between age and dissociation $(P=0.006)$ indicating that dissociation decreased as age increased. The Spearman correlation test indicated no significant relationship between education and dissociation $(P=0.087)$. Regression analysis showed the highest correlation to be between dissociation and age, followed by education $\left(R^{2}=0.154\right)$. There was no significant relationship found between the level of disappointment and DES score $(P=0.269)$.

Table 1 compares the independent t-test scores for mean DES and disappointment between the married and single groups. There was no significant difference between groups for mean DES $(P=0.139)$. A significant difference for mean disappointment score was found between groups $(P=0.047)$. Table 2 compares the independent $t$ test results for mean DES between males and females. No significant difference was observed between groups for mean dissociation score $(P=0.064)$.

One-way ANOVA (Table 3 and Table 4 ) shows a significant relation between means DES score and occupation $(P=$ 0.027). There was a significant relationship between DES 
Dissociative Experiences and Attempting Suicide

Table 1. Comparison of Average DES and Disappointment Scores in Married and Single Individuals With Attempted Suicide

\begin{tabular}{|c|c|c|c|c|c|c|c|}
\hline Variation & Marital Status & Number & Mean & SD & $T$ Test & $d f$ & $P$ Value \\
\hline \multirow{2}{*}{ DES } & Single & 26 & 25.5 & 13.85 & 1.494 & 96 & 0.139 \\
\hline & Married & 72 & 20.4 & 15.34 & 1.56 & 48.69 & 0.123 \\
\hline \multirow{2}{*}{ Disappointment score } & Single & 26 & 10.88 & 3.19 & -2.01 & 96 & 0.047 \\
\hline & Married & 72 & 12.3 & 3.05 & -1.96 & 42.59 & 0.056 \\
\hline
\end{tabular}

Table 2. Comparison of Mean DES Scores Between Males and Fmales

\begin{tabular}{ccccccc}
\hline Gender & Number & Mean & SD & T Test & $\boldsymbol{d f}$ & 96 \\
Male & 40 & 18.5 & 13.06 & -1.873 & -1.94 & 9.064 \\
Female & 58 & 24.08 & 15.99 & 0.057 & 93.17 \\
\hline
\end{tabular}

and being employed $(P=0.017)$ or self-employed $(P=$ $0.013)$, but no relationship between DES and being a housewife, retired or a student. Being employed showed a significant relationship with being a housewife $(P=0.03)$ and being self-employed showed a significant relationship with being a housewife $(P=0.018)$. Being retired or a student showed no significant relationships.

Table 5 shows that according to correlation testing, there was no significant relationship between disappointment and dissociative experiences. Neither of them affected the occurrence of the other $(P=0.933)$. There was a significant relationship between age and dissociation $(P=0.006)$ in which dissociation decreased as age increased. There was also no significant relationship between the level of education and dissociation $(P=0.087)$.

\section{Discussion}

This cross-sectional study was performed to investigate the prevalence and extent of dissociative experiences and assess its relationship with attempted suicide. It was assumed that suicide attempters should exhibit a higher and more severe prevalence for these experiences than the general population. In the study population, the mean dissociative experience scores as measured by the DES standard questionnaire was 21.7. The criteria for the analysis of DES indicates that this is a high score and beyond normal range. ${ }^{18}$

No study has been conducted on the general population in Iran, but meta-analysis conducted by van IJzendoorn and Schuengel ${ }^{19}$ evaluated 11 studies in the natural population and showed an average score of 11.57 . Firoozabadi et $\mathrm{al}^{20}$ tested 160 patients hospitalized in a psychiatric section reported an average score of 22.95 , which is not significantly different from the results of the present study. Both the present study and Firoozabadi et $\mathrm{al}^{20}$ found no difference in mean scores for dissociation between males and females, which consistent with the results of Sar et al. ${ }^{21}$ It is clear from Firoozabadi et $\mathrm{al}^{20}$ that there is a relationship between a high dissociation score and the severity of mental disorder and the presence of personality traits of emotionality and impulsiveness.

The level of disappointment in studies was considered to be a sign of the severity of depression and suicidal
Table 3. Comparison of mean DES by Occupation ${ }^{a}$

\begin{tabular}{lccc}
\hline Occupation & Number & Mean & SD \\
\hline Unemployed & 11 & 29.7 & 13.95 \\
Employed & 17 & 16.1 & 15.13 \\
Self-employed & 24 & 16.3 & 12.62 \\
Retired & 5 & 15.9 & 20.94 \\
Student & 7 & 26.6 & 9.49 \\
Housewife & 34 & 25.6 & 15.22 \\
Total & 98 & 21.7 & 15.6 \\
\hline a $F$ value $=2.66, P=0.027$ & & &
\end{tabular}

Table 4. Post hoc (LSD) Comparison of DES Scores by Occupation

\begin{tabular}{lccccc}
\hline \multirow{2}{*}{ DES } & \multicolumn{5}{c}{ ANOVA } \\
\cline { 2 - 6 } & Sum of Squares & $\boldsymbol{d f}$ & Mean square & $\boldsymbol{F}$ & $\boldsymbol{P}$ \\
\hline Between groups & 2784.310 & 5 & 556.862 & 2.664 & .027 \\
Within groups & 19230.188 & 92 & 209.024 & & \\
Total & 22014.498 & 97 & & & \\
\hline
\end{tabular}

Table 5. Correlation Between Beck's Questionnaire Score and DES Score, Age and Education

\begin{tabular}{ll}
\hline Analysis & Variant \\
\hline Correlation coefficient $(\mathrm{R})=0.009 ; P=0.933$ & Beck disappointment scale \\
Correlation coefficient $(\mathrm{R})=-0.278 ; P=0.006$ & age \\
Correlation coefficient $(\mathrm{R})=-0.174 ; P=0.087$ & education level \\
\hline
\end{tabular}

tendency. Most of these people did not intend to commit suicide, but when combined with the relative high score for dissociation, it can be concluded that suicidal behavior in these individuals was an impulsive decision rather than the result of long-term depression.

The average disappointment score in these individuals was 11.92 , which is in the normal range. No significant relationship was found between the degree of dissociation and level of disappointment, which confirms this view. Significant differences were observed in the disappointment scores of married and single groups. A higher level of disappointment among married couples suggests that is may be a factor in mental pressure that prompts a decision for an impulsive suicide attempt resulting from disagreement and family conflict. There also was no significant difference between level of disappointment and DES score and no significant differences between the level 
of education and DES score.

Marital status did not affect the DES score. In previous studies, the relationship between dissociation, education and marital status was not been investigated, so there was no basis for comparison. There was a significant difference between the level of dissociation and employment status, with the highest score belonging to the unemployed, but no relationship shown to being a housewife, retired or a student. Being employed had a significant relationship only with being a housewife. Being self-employed only showed a significant relationship with being a housewife. Being retired or a student showed no significant relationships.

Sar et $\mathrm{al}^{22}$ described the influence of the level of culture on dissociative disorders. If the value placed on an individual by the culture is commensurate with the occupation, this significant difference is justifiable. Linear regression analysis shows that age is partially predictive of a high dissociation score. This indicates that, if age is controlled for, the difference between the disorders will be eliminated. The final diagnosis of some patients was unclear because of insufficient hospitalization (shortage of beds in hospitals) or the short duration of hospitalization; thus, the relationship between the final diagnosis and the suicide attempt could not be determined. No significant differences were found for level of education and DES score. Marital status did not affect the quality of life.

Previous studies have not investigated the relationship between dissociation and educational or marital status; thus, there was no basis available for comparison. There was a significant difference between the level of dissociation and occupation, with the highest score recorded by the unemployed. There was a significant relationship between having and not having a job, but this was not related to being a housewife, retired or a student. Additionally, education level and sex were only slightly predictive of a high score of dissociation in patients. Age showed the highest relationship with dissociation, with a decrease in the prevalence of dissociative experiences with an increase in age.

Investigations have shown that one of the strong factors in anticipating these dissociative experiences is a history of traumatic childhood events. It can be said that the diagnosis may not be highly accurate and, if a more accurate examination is made, some of these patients, especially those who are categorized as having severe psychiatric disorders and high DES scores, may have a dissociative disorder and a positive history of traumatic childhood experiences. In other words, this could be defined as a subset for each disorder based on traumatic childhood experience. The attention given to a subset in these disorders can lead to better management and treatment of these conditions. Van IJzendoorn and Schuengel ${ }^{19}$ showed that those who were exposed to abuse during childhood had a high score for dissociative experiences.

\section{Conclusion}

The present study found no significant difference between

\section{Research Highlights}

What Is Already Known?

Although it is common for patients with dissociative disorder to report a history of suicide attempts, there is very little data systematically comparing suicidality in patients with dissociative disorders versus patients without these disorder. Suicide risk in dissociative identity disorder (DID) is a significant concern. People With DID have one of the highest risks for suicide. Suicide in DID can become complicated when different alters are involved.

\section{What This Study Adds?}

The present study found no significant difference between suicidal tendency and the level of disappointment and dissociative symptoms.

suicidal tendency and the level of disappointment and dissociative symptoms. One reasons for this is the low study population size. Another reason, as mentioned earlier, is that these people may not have the actual intention to commit suicide. The reason for their referral to a psychiatric center was caused by an impulsive decision originating from a periodic stressor, rather than from psychopathology and a psychiatric disorder.

\section{Authors' Contributions}

All authors contributed equally to this study.

\section{Conflict of Interest Disclosures}

The authors declare that they have no conflicts of interest.

\section{Ethical Approval}

Ethical approval for this study was obtained from the Shiraz University of Medical Sciences Ethics Board.

\section{References}

1. Dell PF, O'Neil JA. Preface. In: Dell PF, O'Neil JA. Dissociation and the Dissociative Disorders: DSM-V and Beyond. Routledge; 2010.

2. Rodriguez AH, Caldera T, Kullgren G, Renberg ES. Suicidal expressions among young people in Nicaragua: a communitybased study. Soc Psychiatry Psychiatr Epidemiol. 2006;41(9):692697. doi:10.1007/s00127-006-0083-x.

3. Dell PF. A new model of dissociative identity disorder. Psychiatr Clin NorthAm.2006;29(1):1-26, vii.doi:10.1016/j.psc.2005.10.013.

4. Butler LD, Duran RE, Jasiukaitis P, Koopman C, Spiegel D. Hypnotizability and traumatic experience: a diathesis-stress model of dissociative symptomatology. Am J Psychiatry. 1996;153(7 Suppl):42-63. doi:10.1176/ajp.153.8.A42.

5. Gleaves DH, May MC, Cardena E. An examination of the diagnostic validity of dissociative identity disorder. Clin Psychol Rev. 2001;21(4):577-608.

6. Dell PF. The multidimensional inventory of dissociation (MID): A comprehensive measure of pathological dissociation. J Trauma Dissociation. 2006;7(2):77-106. doi:10.1300/J229v07n02_06.

7. Weiten W, Lloyd MA, Dunn DS, Hammer EY. Psychology Applied to Modern Life: Adjustment in the 21st Century. 9th ed. Wadsworth Cengage Learning; 2008. 
8. Snyder CR. Coping: The Psychology of What Works. New York: Oxford University Press; 1999.

9. Zeidner M, Endler NS. Handbook of Coping: Theory, Research, Applications. New York: John Wiley Sons; 1996.

10. Salter AC. Hilary Eldridge. Transforming Trauma: A Guide to Understanding and Treating Adult Survivors of Child Sexual Abuse. Sage Publications Inc; 1995:220.

11. Myers JE, Berliner L, Briere J, Hendrix CT, Jenny C, Reid TA. The APSAC Handbook on Child Maltreatment. 2nd ed. Sage Publications; 2002:63.

12. Bidaki R, Shirani S, Shamsian $M$, et al. A Review of the Various Suicide Methods Used Around the World. Int J Med Rev. 2016;3(4):504-507. doi:10.15171/ijmr.2016.11.

13. Briere J. Dissociative symptoms and trauma exposure: specificity, affect dysregulation, and posttraumatic stress. J Nerv Ment Dis. 2006;194(2):78-82. doi:10.1097/01.nmd.0000198139.47371.54.

14. Sajadi SF, Arshadi N, Zargar Y, Honarmand MM, Hajjari Z. Borderline personality features in students: the predicting role of schema, emotion regulation, dissociative experience and suicidal ideation. Int J High Risk Behav Addict. 2015;4(2):e20021. doi:10.5812/ijhrba.20021v2.

15. Merckelbach H, Muris P. The causal link between self-reported trauma and dissociation: a critical review. Behav Res Ther. 2001;39(3):245-254. doi:10.1016/S0005-7967(99)00181-3.
16. Snow MS, Beckman D, Brack G. Results of the dissociative experiences scale in a jail population. Dissociation. 1996;9(2):98103.

17. Bernstein EM, Putnam FW. Development, reliability, and validity of a dissociation scale. J Nerv Ment Dis. 1986;174(12):727-735.

18. Ross CA, Halpern N. Trauma Model Therapy: A Treatment Aproach for Trauma, Dissociation and Complex Comorbidity. Greenleaf Book Group; 2009:485.

19. van ljzendoorn MH, Schuengel C. The measurement of dissociation in normal and clinical populations: Meta-analytic validation of the Dissociative Experiences Scale (DES). Clin Psychol Rev. 1996;16(5):365-382. doi:10.1016/0272-7358(96)00006-2.

20. Firoozabadi A, Abedi Z, Aliyari R, Zolfaghari B, Ghanizadeh A. Psychometric characteristics of the Persian (Farsi) version of attachment style questionnaire. Iran J Med Sci. 2014;39(6):506514.

21. Sar V, Tutkun H, Alyanak B, Bakim B, Baral I. Frequency of dissociative disorders among psychiatric outpatients in Turkey. Compr Psychiatry. 2000;41(3):216-222. doi:10.1016/s0010440x(00)90050-6.

22. Sar V, Kundakci T, Kiziltan E, et al. The Axis-I Dissociative Disorder Comorbidity of Borderline Personality Disorder Among Psychiatric Outpatients. J Trauma Dissociation. 2003;4(1):119-136. doi:10.1300/J229v04n01_08. 